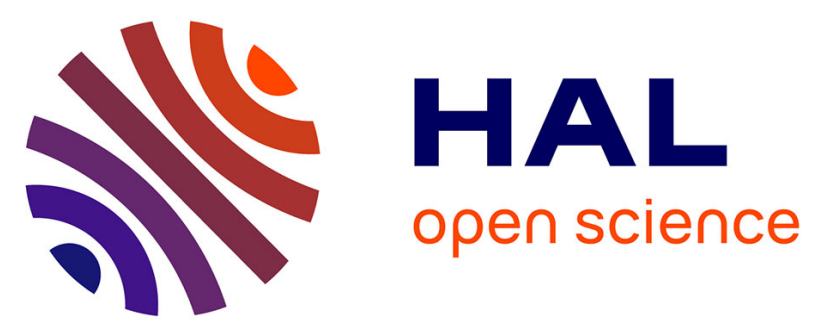

\title{
Le projet européen AIMWATER : utilisation de l'humidité superficielle des sols mesurée par radar embarqué (ERS/SAR) pour la modélisation pluie-débit
}

A. Weisse, F. Le Hegarat, D. Aubert, C. Loumagne

\section{- To cite this version:}

A. Weisse, F. Le Hegarat, D. Aubert, C. Loumagne. Le projet européen AIMWATER: utilisation de l'humidité superficielle des sols mesurée par radar embarqué (ERS/SAR) pour la modélisation pluiedébit. La Houille Blanche - Revue internationale de l'eau, 2002, 1, pp.35-40. 10.1051/lhb/2002003 . hal-01098283

\section{HAL Id: hal-01098283 \\ https://hal.science/hal-01098283}

Submitted on 7 Jan 2015

HAL is a multi-disciplinary open access archive for the deposit and dissemination of scientific research documents, whether they are published or not. The documents may come from teaching and research institutions in France or abroad, or from public or private research centers.
L'archive ouverte pluridisciplinaire $\mathbf{H A L}$, est destinée au dépôt et à la diffusion de documents scientifiques de niveau recherche, publiés ou non, émanant des établissements d'enseignement et de recherche français ou étrangers, des laboratoires publics ou privés. 


\title{
Le projet européen AIMWATER : utilisation de l'humidité superficielle des sols mesurée par radar embarqué (ERS/SAR) pour la modélisation pluie-débit
}

\author{
The European AIMWATER project : using surface soil moisture monitoring \\ from ERS/SAR for the rainfall-runoff modelisation
}

\author{
par Anne Weisse*1 ${ }^{* 1}$ Sylvie Le Hégarat**, David Aubert*, Cécile Loumagne* \\ * Cemagref, ** CETP/CNRS
}

Soil moisture is a key hydrological variable in flood forecasting, because it determines the partition of the rain between runoff and infiltration and thus governs the flows at the outlet of a catchment. The aim of the AIMWATER project is to improve the commonly used hydrological tools in an operational context for reservoir management by assimilating soil moisture data into rainfall-runoff models. The surface soil moisture is derived from ERS/SAR spaceborne data at basin scale. The project has validated the methodology set up to derive the spaceborne information showing its consistency both in time ( 3 years of consecutive data) and in space ( 3 Seine sub-catchments). Different assimilation methodologies have been put forward for use and improved in a forecasting context : the variational method is presented here. It updates the parameters values of hydrological models during the forecast. We present in this paper the results of these methodologies applied on the Seine sub-catchments.

\section{INTRODUCTION}

L'humidité superficielle des sols est une variable clé en hydrologie : elle détermine l'évaporation réelle, la répartition de la pluie entre infiltration et ruissellement et l'infiltration vers les couches de sol profondes. L'intérêt de prendre en compte dans les modèles hydrologiques, une information telle que l'humidité de surface ou des couches profondes des sols par l'intermédiaire de variables mesurables, a été étudié par plusieurs équipes de recherche $[1,2,3]$. Ces travaux ont montré que malgré les progrès réalisés en modélisation hydrologique au cours des dernières décennies, les modèles présentent de nombreuses limitations pour la prévision des débits à l'exutoire du bassin qui pourraient être franchies en intégrant un nouveau type d'information sur l'état hydrique des bassins. Dans le cadre d'une approche de modélisation globale des bassins versants, l'humidité des sols prise en compte doit être représentative du bassin versant dans son entier, dès lors, l'utilisation de la télédétection s'impose comme étant le seul outil permettant de caractériser instantanément des zones étendues. La télédétection radar a montré son intérêt pour le suivi de l'humidité superficielle au cours du cycle hydrologique en donnant une information spatialisée, représentative à l'échelle du bassin versant.

' DIREN Lorraine, Service Hydrologie et Annonce de Crues - Cellule Etudes, 19 av Foch, BP 60223, 57005 Metz Cedex 1 .

e-mail : anne.weisse@lorraine.environnement.gouv.fr
Cet article présente les méthodes développées dans le cadre d'un projet financé par la commission européenne pour encourager l'utilisation de données d'observation de la Terre, le projet AIMWATER (Analysis, Investigation and Monitoring of WATER resources, for the management of multipurpose reservoirs). Ce projet a été proposé dans le but de montrer les possibilités d'amélioration des outils de prévision hydrologique en s'appuyant sur l'intégration de données de télédétection dans des modèles communément utilisés à cette fin.

En premier lieu, il s'agit de montrer comment on peut utiliser les données du radar à synthèse d'ouverture (SAR) du satellite européen ERS pour déterminer des indices hydriques superficiels à l'échelle de bassins versants : une méthodologie [4] a été mise au point afin d'identifier des cibles (types d'occupation du sol) dites «sensibles", sur lesquelles le signal radar rétrodiffusé dépend fortement de l'humidité du sol et qui sont choisies pour une estimation pertinente de l'état de surface.

La deuxième étape consiste à montrer comment on peut améliorer la modélisation pluie-débit et plus particulièrement la prévision des crues en utilisant cette nouvelle donnée, c'est-à-dire par assimilation dans un modèle hydrologique de l'humidité du sol issue de l'information radar. Les méthodes actuellement utilisées sont de deux types et s'appuient sur des travaux récents $[5,6]$. La première, qui est une méthode de prévision de crues, est dite variationnelle, et consiste à modifier le jeu de paramètres du modèle de manière à mieux 


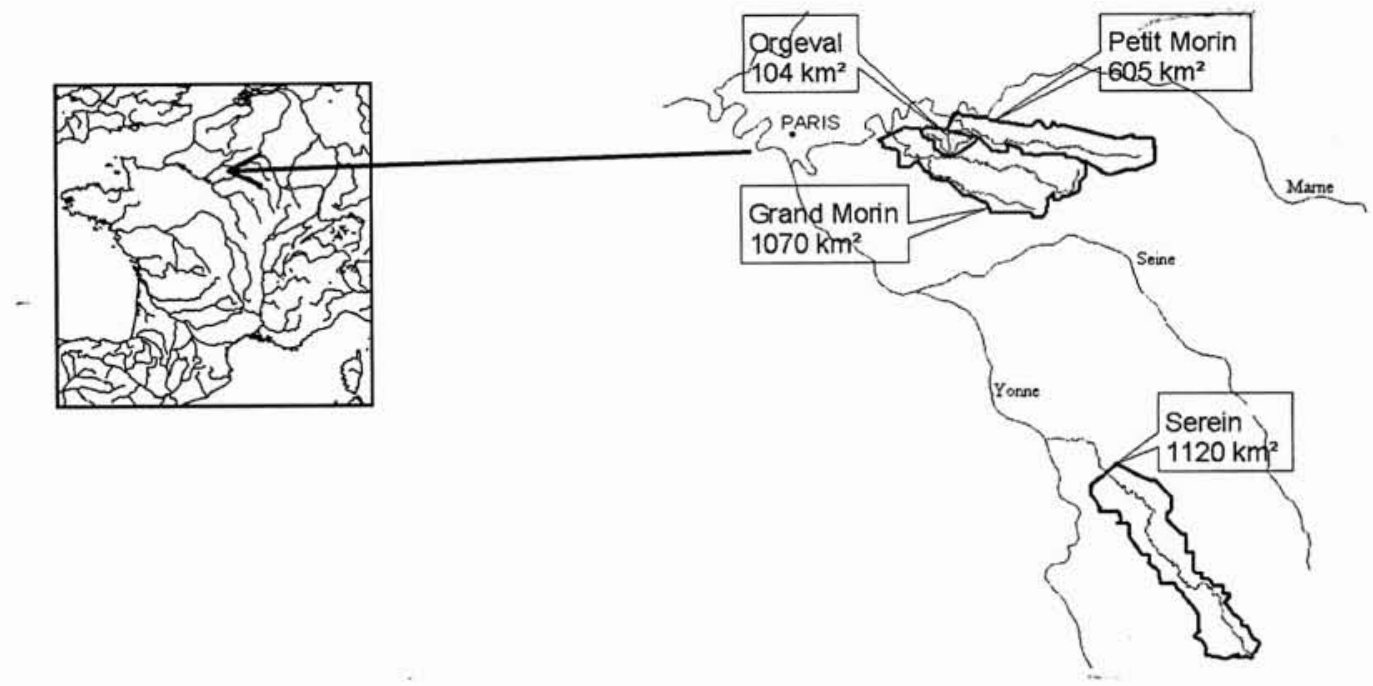

Figure 1 : Localisation des bassins versants étudiés.

simuler les débits lors de la prévision. Les données d'humidité du sol sont utilisées pour améliorer la simulation des débits en s'approchant au plus près de la réalité. La méthode séquentielle [7] consiste à corriger les variables internes du modèle lorsque des observations d'humidité sont disponibles afin de réduire l'écart entre les valeurs mesurées et simulées par le modèle. Pour ne pas trop perturber le modèle et prendre en compte les erreurs sur les observations, cette correction n'est que partielle, le facteur de correction étant calculé à chaque pas de temps par l'intermédiaire d'un filtre de Kalman étendu. Ce sont les résultats de la méthode variationnelle qui sont présentés dans la suite.

Le but final du projet est de fournir des outils opérationnels de prévision de crue aux gestionnaires de barrages réservoirs (IIBRBS Institut Interdépartemental des Barrages Réservoirs du Bassin de la Seine, ARBSLP Associaçao de regantes e beneficiarios de Silves association d'irriguants portugais). En particulier, pour la France, des sous bassins versants de la Seine ont été choisis pour mettre au point la méthodologie d'assimilation de données satellitales (cf. fig. 1): le Grand Morin $\left(1190 \mathrm{~km}^{2}\right)$, l'Orgeval $\left(104 \mathrm{~km}^{2}\right)$, qui est un sous bassin versant du Grand Morin, le Petit Morin $\left(605 \mathrm{~km}^{2}\right)$ et le Serein $\left(1120 \mathrm{~km}^{2}\right)$. Ces bassins versants sont principalement agricoles, avec un relief peu marqué.

\section{II — ESTIMATION DES DONNÉES D'HUMIDITÉ DES SOLS À L'ÉCHELLE D'UN BASSIN VERSANT À PARTIR DE DONNÉES ERS/SAR}

\section{II.1 Méthodologie}

L'estimation de l'état hydrique du sol à partir de données ERS/SAR repose sur le fait que le signal mesuré est fonction de la constante diélectrique $\varepsilon$ de la surface imagée, $\varepsilon$ dépendant elle-même du contenu en eau du sol. En outre, la longueur d'onde du radar SAR étant $\lambda \simeq 5,6 \mathrm{~cm}$ (bande $\mathrm{C}$, fréquence $\mathrm{f} \simeq 5,3 \mathrm{GHz}$ ), l'indice hydrique obtenu est représentatif de l'humidité des sols de la couche superficielle, c'est-à-dire des 5-10 premiers centimètres de sol.

Outre $\varepsilon$, la géométrie de la surface et son couvert végétal influent également sur le signal rétrodiffusé. Il convient donc de s'affranchir de ces deux effets avant de pouvoir relier le signal mesuré à l'humidité superficielle. Dans un premier temps, une carte d'occupation des sols est obtenue par classification non supervisée [8] combinant des images multispectrales LANDSAT/TM et multitemporelles ERS/SAR. Cette carte, à la résolution spatiale des images ERS $\left(25 \times 25 \mathrm{~m}^{2}\right)$, est utilisée pour sélectionner les cultures ou cibles pour lesquelles le signal peut être exploité (pour l'obtention de nos indices hydriques). Sur ces zones, le signal SAR est «corrigé » de l'effet de la végétation quand celle-ci est présente et non dominante, cette deuxième condition excluant notamment les cas de végétation dense (forêt, céréales à maturité....). Par ailleurs, à l'heure actuelle, le modèle de transfert radiatif utilisé pour modéliser la végétation n'a été validé que dans le cas des céréales d'hiver (ce qui exclut les autres cultures sauf en période de sol nu). Ce traitement conduit alors, pour chaque date, à l'obtention d'une image d'un signal SAR « équivalent sol nul ", image comportant des zones plus ou moins étendues de données manquantes (correspondant aux cas où le signal SAR n'ayant pu être corrigé ne sera pas exploité).

Ensuite, pour s'affranchir de l'effet de la rugosité, l'approche choisie est de moyenner le signal sur une région suffisamment large pour que l'on puisse supposer un effet de la rugosité approximativement constant tout au long de l'année : dans le cas d'un site agricole, si l'on considère une variété suffisamment large de cultures, les travaux du sol n'ayant pas lieu aux mêmes périodes de l'année, cette hypothèse est vérifiée empiriquement.

Finalement la méthodologie consiste en :

- la sélection, pour chaque date, des cibles (cultures ou types d'occupation du sol) sur lesquelles le signal SAR « équivalent sol nu » pourra être estimé ;

- la correction, pour ces cibles et à la date considérée, des effets de la végétation ;

- le filtrage de l'effet des changements de la rugosité par moyennage sur le plus grand nombre possible de cibles ;

- la conversion du signal SAR moyen corrigé ainsi obtenu $\tilde{\boldsymbol{\sigma}}_{0}$ en une humidité volumique superficielle par le biais d'une relation linéaire empirique (entre $\tilde{\boldsymbol{\sigma}}_{0}$ en $\mathrm{dB}$ et $\mathrm{W}_{\mathrm{v}}$ en $\%$ ). 


\section{- II.2 Résultats pour les bassins versants étudiés}

Pour chacun des bassins versants étudiés (cf. fig. 1), un ensemble d'images a été acquis : de novembre 1995 à octobre 1998 pour l'Orgeval (programme PNTS de l'ESA) et de janvier 1999 à avril 2001 pour le Grand Morin, le Petit Morin et le Serein (projet AIMWATER). Concomitantes à chaque passage du satellite ERS, des campagnes de vérité terrain ont été organisées pour mesurer l'humidité des sols par la méthode gravimétrique (Orgeval et Serein), en addition des appareils de mesure de l'humidité des sols (type TDR) installés sur l'Orgeval et le Serein fournissant des données en continu (pas de temps d'acquisition de 12 heures et profondeurs de sol allant jusqu'à $160 \mathrm{~cm}$ ). Ces données permettent d'étalonner les relations entre signal $\tilde{\boldsymbol{\sigma}}_{0}(\mathrm{en} \mathrm{dB})$ et vérité terrain $\mathrm{W}_{\mathrm{v}}$ (humidité superficielle pour le bassin versant, en $\mathrm{cm}^{3} / \mathrm{cm}^{3}$ ). Enfin, des mesures de végétation ont également été effectuées en vue des corrections de végétation nécessaires sur le signal SAR.

La figure 2 présente les résultats de l'inversion des données ERS/SAR par la méthode décrite précédemment [9]. Le cas de l'Orgeval permet de conclure que la relation entre le signal $\tilde{\boldsymbol{\sigma}}_{0}$ (en $\mathrm{dB}$ ) et les mesures in situ d'humidité des sols $\mathrm{W}_{\mathrm{v}}$ est relativement stable d'une année à l'autre. La pente de cette relation est à peu près constante pour les trois années étudiées et proche de 0,3 . L'ordonnée à l'origine varie légèrement d'une année à l'autre, ce qui est probablement dû à un changement de l'effet moyen de la rugosité du fait de la rotation des cultures et du changement de direction des sillons dans les parcelles. Enfin, en termes d'inversion, l'erreur sur les estimations de $\mathrm{W}_{\mathrm{v}}$ reste inférieure à $4 \%$ si l'on utilise une relation établie sur des années précédentes pour inverser les données de l'année en cours. Les résultats sur les trois autres bassins versants (Grand Morin, $1190 \mathrm{~km}^{2}$, Petit Morin, $605 \mathrm{~km}^{2}$, et Serein, $1120 \mathrm{~km}^{2}$ ), qui sont bien plus étendus que le bassin versant de l'Orgeval $\left(104 \mathrm{~km}^{2}\right)$, sont présentés dans [10]. Les conclusions sont les mêmes que précédemment: bonne corrélation entre $\tilde{\boldsymbol{\sigma}}_{\mathrm{o}}(\mathrm{en} \mathrm{dB})$ et les mesures in situ de $\mathrm{W}_{\mathrm{v}}$ (coefficients $\mathrm{R}^{2}$ sont proches de 0,9 ), stabilité de la pente des relations (proche de 0,3 ), erreurs inférieures à $4 \%$.

Ces résultats sont très encourageants, puisqu'ils montrent que la méthodologie mise au point pour l'Orgeval [4] s'applique à d'autres bassins versants agricoles. En effet, la pente de la relation $\tilde{\boldsymbol{\sigma}}_{0}(\mathrm{~dB})=\mathrm{f}\left(\mathrm{W}_{\mathrm{v}}(\%)\right)$ est indépendante du bassin versant, et de même celle de la relation $\mathrm{W}_{\mathrm{v}}(\%)=\mathrm{g}\left(\tilde{\boldsymbol{\sigma}}_{0} \mathrm{~dB}\right)$, non représentée sur les figures, mais qui est celle effectivement utilisée lors de l'inversion. Ainsi, pour étudier un nouveau bassin versant, une pente par défaut $(=2,8)$ peut être utilisée pour inverser le signal radar pendant une première année de calage de la relation.

Les principales limites d'application de la méthode seront alors : la présence de zones de végétation dense sur le bassin, ou de fort relief, ou enfin de rugosité non stable même à l'échelle du bassin versant.

\section{III — ASSIMILATION DE DONNÉES D'HUMIDITÉ DES SOLS DANS UN MODÈLE PLUIE-DÉBIT POUR LA PRÉVISION DE CRUE : LA MÉTHODE VARIATIONNELLE}

Les modèles hydrologiques sont trop imprécis pour être utilisés tels quels dans une opération de prévision : il y a généralement un écart important entre les résultats du modèle et l'observation. Or si le modèle s'éloigne trop des observations à un moment donné, les prévisions auront peu de chance d'être fiables. L'amélioration des performances des modèles en prévision nécessite donc en premier lieu une correction de la trajectoire du modèle pour en limiter la divergence. $\mathrm{Ce}$ contrôle de la dynamique de l'évolution du système hydrologique modélisé se fait par mise à jour des états internes et de paramètres du modèle grâce à l'assimilation de données externes au modèle. La méthode d'assimilation proposée ci-dessous vise à utiliser aussi bien une information récente sur les débits qu'une information sur l'humidité des sols pour la prévision. C'est l'intérêt de ce nouveau type de données utilisé comme variable prédictive en assimilation qui a fait l'objet de nos recherches dans ce domaine.

Les résultats présentés ont été obtenus en utilisant des données journalières d'humidité des sols (obtenues par des appareils TDR). L'hypothèse faite est que la mesure ponctuelle est représentative de la variabilité temporelle des humidités sur l'ensemble des bassins versants.

\section{- III.1 Méthodologie}

Le but de la démarche est d'étudier l'intérêt d'introduire l'humidité du sol dans une méthode de mise à jour des paramètres basée sur l'assimilation des débits [11]. La méthode initiale a été testée avec des modèles conceptuels de type GR4j [12] et GRHum [13] et a montré de bonnes performances dans l'amélioration des prévisions de crue [14].

La méthodologie développée pour prendre en compte l'humidité des sols a déjà été exposée en détail $[15,16]$ et seules les grandes lignes sont rappelées ici.

Le principe de la méthode consiste à mettre à jour les valeurs des paramètres du modèle en utilisant les observations de débit disponibles sur une période antérieure au jour de prévision. Il s'agit de réduire la différence entre les débits observé et simulé le jour de la prévision de manière à mieux prévoir les débits à venir. Une première correction sur les débits simulés est effectuée avant toute opération de mise à jour. Elle est basée sur une analyse préliminaire des erreurs de débits simulés. Ensuite, on suppose que l'on peut pallier les écarts récents entre modèle et observation en modifiant provisoirement le jeu de paramètres du modèle, pour mieux s'adapter à la situation en cours. Ces modifications sont effectuées sur une période de rétroaction longue (de l'ordre de 60 jours), de manière à ce qu'elles aient un impact réel sur le débit simulé ce jour.

Nous avons montré que l'humidité des sols est liée à certains états du système du modèle hydrologique utilisé. Par exemple, le stock d'eau contenu dans les premiers 10 centimètres du sol peut expliquer $80 \%$ de la variance du contenu en eau du réservoir sol de GR4j, par une relation linéaire. On cherche donc à utiliser cette information externe pour améliorer la simulation des débits de façon plus vraisemblable, c'est-à-dire en rendant mieux compte des observations récentes d'humidité de surface. Il y a un grand nombre de jeux de paramètres qui permettent de minimiser les dernières erreurs sur les débits. Le but de la démarche est de voir si une information sur l'humidité des sols peut aider à choisir une solution qui serait plus proche de la réalité tant au niveau des débits que des humidités et qui serait donc plus performante que méthode initiale. L'approche choisie consiste à ajouter 
ORGEVAL
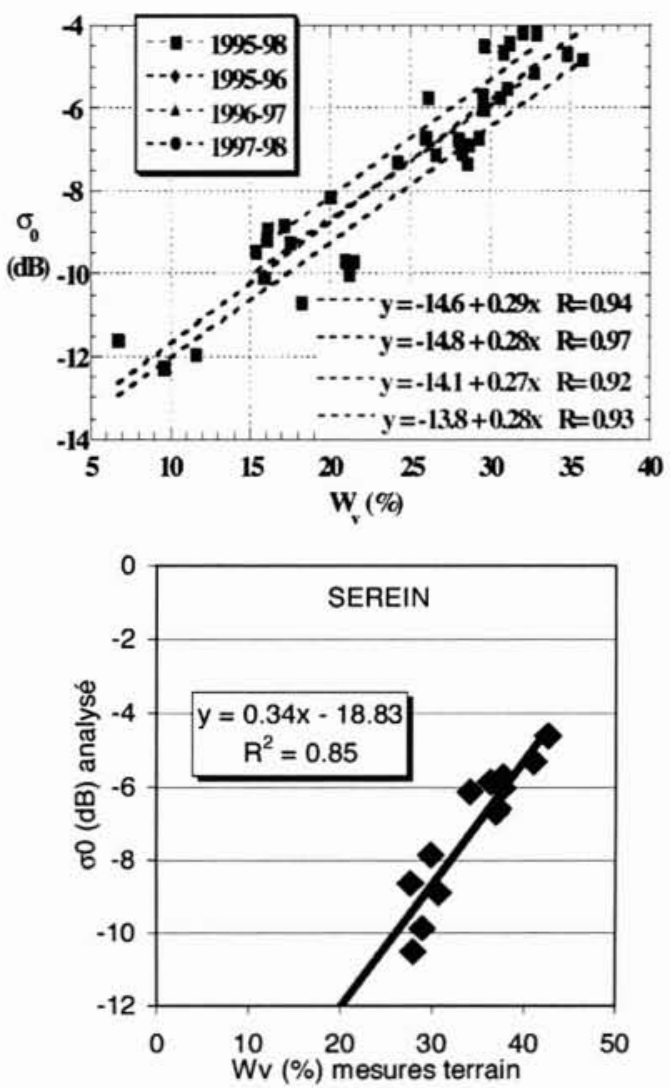
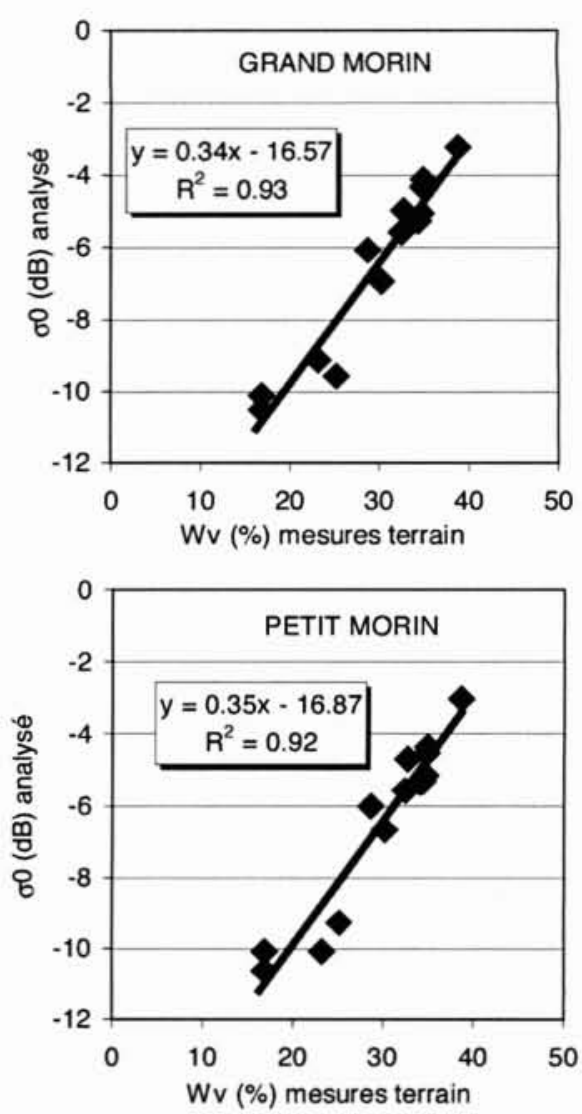

Figure 2: Relation entre le signal ERS/SAR analysé $\sigma_{o}(\mathrm{~dB})$ et l'humidité volumétrique des sols $\mathrm{W}_{\mathrm{v}}(\%)$ pour les différents bassins versants étudiés.

une contrainte sur les humidités observées et modélisées par les variables internes des modèles pluie-débit :

$$
\frac{1}{n} \sqrt{\sum_{i=1}^{n}(H o(i)-H c(i))^{2}}<\frac{\sigma}{k}
$$

où $H o$ représente les humidités observées, $H c$ les humidités modélisées, $n$ est le nombre de valeurs d'humidités prises en compte, $\sigma$ l'écart type de $H c$ et $k$ un paramètre qui impose la sévérité de la contrainte (si $k$ est petit, la contrainte est nulle et plus $k$ est grand, plus elle est prise en compte). La valeur de $k$ donne une mesure de l'intérêt de la prise en compte de l'humidité.

La méthodologie s'appuie donc sur deux contraintes : (i) le nouveau jeu de paramètres doit réduire les dernières erreurs sur les débits (ii) ce jeu doit satisfaire la condition (1), sinon il est rejeté.

Le critère de qualité de la prévision choisi est le critère de persistance [17]. Il est évalué pour chaque jour où une prévision est faite (la crue a été définie comme correspondant à un débit supérieur à un seuil de 4 fois le module interannuel), c'est l'erreur quadratique des débits prévus $Q_{\text {prévu }}$ par rapport aux débits réels $Q_{\text {obs }}$ :

$$
\text { Pers }=100\left[1-\frac{\sum_{j}\left(Q_{o b s}(j+L)-Q_{\text {prévu }}(j+L)^{2}\right)}{\sum_{j}\left(Q_{o b s}(j+L)-Q_{o b s}(j)\right)^{2}}\right]
$$

où $j$ est le jour de prévision et $L$ le délai de prévision

\section{- III.2 Résultats pour les bassins versants étudiés}

La méthode a été testée sur les bassins versants du Serein et du Petit Morin avec le modèle GR4j et des données d'humidité TDR. Les données hydro-météorologiques rassemblées sont présentées tableau 1 . Les périodes de test de la méthode correspondent aux périodes de données d'humidité disponibles (cf. tableau 2).

Les résultats de la méthode d'assimilation variationnelle sont présentés en figure 3 .

La méthode de prévision de crue initiale (i.e. utilisant uniquement les débits : $k=0$ ) donne de bons résultats, puisque les coefficients de persistance sont supérieurs à $80 \%$ pour le Serein. Ils varient entre 40 et $50 \%$ pour le Petit Morin.

Ensuite, quand on utilise l'humidité des sols $(k>0)$, les résultats pour le Petit Morin montrent des gains de persistance compris entre 13 et $18 \%$ par rapport à la méthode utilisant uniquement les débits, suivant le délai de prévision et la profondeur de l'humidité prise en compte. La profondeur des humidités prises en compte a peu d'importance : les résultats sont du même ordre de grandeur pour les différentes profondeurs.

Par contre, pour le Serein, l'introduction de l'humidité n'apporte pas d'amélioration importante à la procédure. On gagne au maximum $1,5 \%$ de persistance avec un délai de prévision de 1 jour et $4,2 \%$ avec un délai de 2 jours. L'explication proposée est que la procédure initiale est déjà très performante (Pers $>80 \%$ ), il semble difficile de l'améliorer. 
Tableau I - Données hydro-météorologiques et données d'humidité des sols rassemblées pour les bassins versants.

\begin{tabular}{|l|c|c|c|c|}
\hline & Débit & Pluies & Evapotranspiration & $\begin{array}{c}\text { Humidité des sols } \\
\text { (mesures TDR) }\end{array}$ \\
\hline Pas de temps des données & $24 \mathrm{~h}$ & $24 \mathrm{~h}$ & 10 jours & $\begin{array}{c}12 \mathrm{~h} \\
\text { mais utilisées à 24h }\end{array}$ \\
\hline Serein & Sep. 1955-Fév. 2001 & $\begin{array}{c}\text { Jan. 1955-Fév. } \\
20019 \text { pluviomètres }\end{array}$ & $\begin{array}{c}\text { Moyenne } \\
\text { interannuelle }\end{array}$ & Fév. 1999-Jan. 2001 \\
\cline { 1 - 3 } Petit Morin & Jan. 1970-Jan. 2001 & $\begin{array}{c}\text { Jan. 1970-Fév. } \\
20017 \text { pluviomètres }\end{array}$ & & Sept. 1997-Jan. 2001 \\
\hline
\end{tabular}

Tableau II-Période de test de la méthode et nombre de crues prises en compte.

\begin{tabular}{|l|c|c|c|}
\hline & $\begin{array}{c}\text { Période } \\
\text { de calage }\end{array}$ & $\begin{array}{c}\text { Période } \\
\text { de test }\end{array}$ & $\begin{array}{c}\text { Nombre } \\
\text { de crues }\end{array}$ \\
\hline Serein & $1955-1997$ & $1999-2001$ & 12 \\
\hline Petit Morin & $1970-1995$ & $1997-2001$ & 16 \\
\hline
\end{tabular}

On retiendra que l'utilisation de l'humidité des sols améliore sensiblement la méthode de prévision de crues envisagée, si cette dernière a des performances moyennes pour le bassin versant d'étude.

\section{CONCLUSION}

Le projet AIMWATER a permis de mettre en évidence différents résultats, intéressants pour des gestionnaires de barrages-réservoirs, puisqu'ils peuvent leur permettre d'amé- liorer leur gestion quotidienne, grâce à des outils de prévision de débits pertinents.

Pour ce qui concerne l'inversion de données ERS/SAR en indices hydriques de bassin, cette étude a permis de valider une méthodologie mise au point pour un petit bassin versant, l'Orgeval. Nous avons montré sa robustesse temporelle avec trois années de données consécutives et sa robustesse spatiale avec des résultats sur trois autres bassins versants de tailles plus importantes et de pédologies différentes.

Une méthode d'assimilation variationnelle des données d'humidité a été présentée. Elle peut être utilisée quelle que soit la fréquence temporelle de mesures d'humidité et avec à n'importe quel modèle pluie-débit, pour la prévision de crues. Elle consiste à faire varier le jeu de paramètres du modèle lors de la prévision, en rendant mieux compte des débits et des humidités récemment observés. Elle donne de bons résultats avec des données ponctuelles et journalières d'humidité pour 2 bassins versants tests. Il reste à la tester sur d'autres bassins versants avec des données spatialisées issues de la télédétection. La répétitivité des données (actuellement une image ERS/SAR tous les 35 jours) est aussi un paramètre à prendre

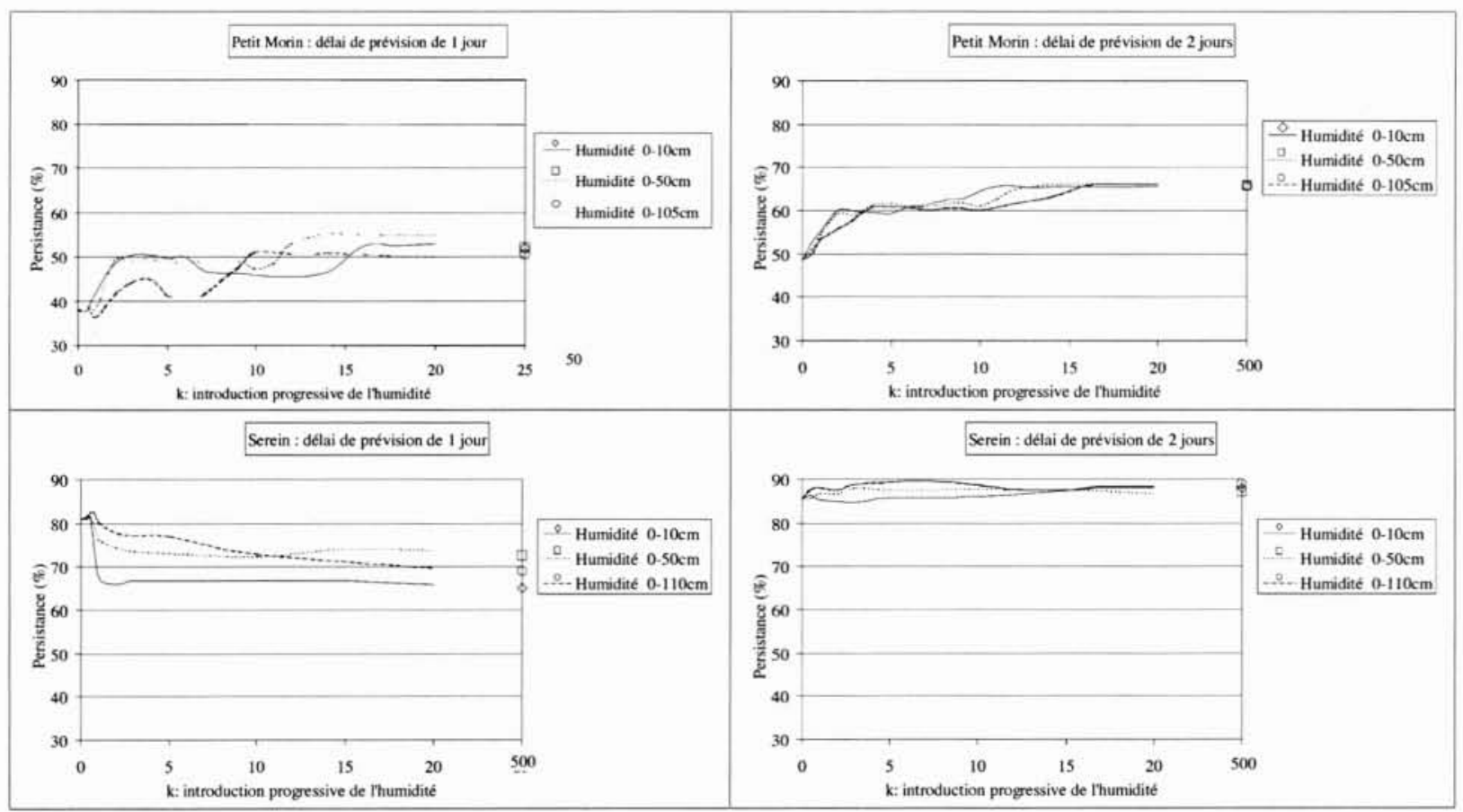

Figure 3 : Assimilation de données d'humidité des sols (données TDR) par une méthode variationnelle de prévision de crue pour ( $k=0$ assimilation des débits, $k>0$ assimilation des débits et des humidités). 
en compte : quelle serait la période de passage optimale à des fins hydrologiques?

Enfin, l'intérêt d'une méthode basée sur le filtre de Kalman par rapport à la méthode variationnelle est à établir pour l'ensemble des bassins versants tests. C'est le but final du projet AIMWATER

\section{REMERCIEMENTS}

Cette étude a été réalisée dans le cadre d'un projet européen financé pour 3 ans (Contract n'ENV4-CT98-0740 (DG12-ESCY)). Les auteurs veulent remercier ici tous les partenaires du projet (Cemagref, CETP/CNRS, Univ. of Valencia, Univ. Independente, IH Wallingford, Ass. Reg. Ben, IIBRBS) et en particulier l'équipe du Cemagref pour son appui technique pour les mesures d'humidité des sols.

\section{BIBLIOGRAPHIE}

[1] Engman E.T. (1990). - Use of microwave remotely sensed soil moisture in hydrologic modeling. In: Application of Remote Sensing in Hydrology, ed. G.W. Kite \& A. Wankiewicz, 279 292. Proc. Symp. No.5, NHRI, Saskatoon, Canada.

[2] Loumagne C., Michel C., Normand M. (1991). - Etat hydrique du sol et prévision des débits. J. Hydrol. 123, 1-17.

[3] Pietroniro A., Soulus E.D., Kouven N., Rotunno O., Muluins D.W. (1993). - Using wide swath C-band SAR imagery for basin soil moisture mapping. Can. J. Remote Sens., Special Issue, January, 77-82.

[4] Quesney A., Le Hégarat-Mascle S., Taconet O., Vidal-Madjar D., Wigneron J.P., Loumagne C., Normand M. (2000). - Estimation of watershed soil moisture index from ERS/SAR data. Remote Sens. Env, 72(3), 290-303.

[5] QuesNeY A. (1999). - Assimilation de données d'humidité de surface dans un modèle hydrologique conceptuel global. Apport de la télédétection radar ERS/SAR. Thèse de docteur, Université de Paris $7,167 \mathrm{p}+$ annexes.

[6] Quesney A., Francois Ch., Ottle C., Le Hegarat S., Loumagne C., Normand M. (2001). - Sequential assimilation of SAR/ERS data in a surface hydric model coupled to a global hydrological model with an extend Kalman filter. Remote sensing and Hydrology 2000, IASH Red Book Publication $n^{\circ} 267$, edited by Brubaker, Ritchie, Rango, ISBN 1-90150246-5 (in press).

[7] Aubert D., Loumagne C., Weisse A., Le Hegarat-Mazcle S. (2001). - Assimilation of Earth Observation data into hydro- logical models: the sequential method. Remote Sensing and Hydrology Symposium, $5^{\text {th }}$ International Workshop on Application of Remote Sensing in Hydrology, octobre 2001, 8p, accepted.

[8] Le Hégarat-Mascle S., Quesney A., Vidal-Madiar D., Taconet O., Normand M., Lotmagne C. (2000). - "Land cover discrimination from multitemporal ERS images and multispectral LANDSAT images: a study case in an agricultural area in France", Int. J. Remote Sens., 21(3), 435-456.

[9] Le Hegarat-Mascle S., Alem F., Quesney A., Normand M., Loumagne C., Weisse A. (2001). - Surface soil moisture monitoring from ERS/SAR data: method and validation over three different watersheds. ISPRS, $8^{\text {th }}$ International Symposium, 8-12 Jan 2001, Aussois, 581-587.

[10] Le Hegarat-Mascle S., Poirier-Quinot M., Alem F., Weisse A., LOUMAGNE C. (2001). - Validation of a methodology to monitor soil moisture from C-band SAR spaceborne in an operational way. Remote Sensing and Hydrology Symposium. $5^{\text {th }}$ International Workshop on Application of Remote Sensing in Hydrology, octobre 2001, 8p, accepted.

[11] YANG X. (1993). - Mise au point d'une méthode d'utilisation d'un modèle pluie-débit conceptuel pour la prévision des crues en temps réel. Thèse de l'Ecole Nationale des Ponts et Chaussées (France), 350 pages.

[12] Eduatno, Nascimento N.O., Yang X., Makhlouf Z., Michel C. (1999). - GR3J : a daily watershed model with three free parameters. Hydrol. Sci. J., 44(2), 263-278.

[13] Loumagne C., Chkir N., Normand M., Ottle C., Vidal-Madjar D. (1996). - Introduction of soil/vegetation/atmosphere continuum in a conceptual rainfall-runoff model. Hydrol. Sci. J. 41(6), 889-902.

[14] YANG X., Michel. C. (2000). - Flood forecasting with a watershed model : a new method of parameter updating. Hydrol. Sci. J., 45(4), 1-10.

[15] Weisse A., Loumagne C., Normand M., Aubert D., Le HegaratMAsCle S., Alem F. (2000). - Assimilation variationnelle de mesures d'humidité de surface dans un but de prévision de crue. Atelier « Modélisation de l'atmosphère 2000 », Toulouse 2930 novembre $2000,31-34$.

[16] Weisse A., Michel C., Aubert D., Loumagne C. (2001). Assimilation of soil moisture in a hydrological model for flood forecasting. Soil-Vegetation-Atmosphere Transfer Schemes and Large-Scale Hydrological Models. Proc. of the $6^{\text {th }}$ IAHS Scientific Assembly at Maastricht, The Netherlands, July 2001, IAHS Publ. no. ${ }^{\circ} 270,249-256$.

[17] Walus J. R., Todist E., (1975). - Comment upon the residual mass curve coefficient. J. Hydrol. 24, 201-205. 